\title{
Identification of Stellar Flares Using Differential Evolution Template Optimization
}

\author{
Kellen D. Lawson ${ }^{1}$ (D), John P. Wisniewski ${ }^{1}$ (D), Eric C. Bellm ${ }^{2}$ (D) Adam F. Kowalski ${ }^{3}$ (D), and David L. Shupe \\ ${ }^{1}$ Homer L. Dodge Department of Physics and Astronomy, University of Oklahoma, 440 W. Brooks Street, Norman, OK 73019, USA \\ ${ }^{2}$ Department of Astronomy, University of Washington, Box 351580, Seattle, WA 98195, USA \\ 3 Astrophysical \& Planetary Sciences, University of Colorado, Boulder, CO 80309, USA \\ ${ }^{4}$ IPAC, California Institute of Technology, 1200 E. California Blvd, Pasadena, CA 91125, USA \\ Received 2019 March 11; revised 2019 June 21; accepted 2019 July 22; published 2019 August 21
}

\begin{abstract}
We explore methods for the identification of stellar flare events in irregularly sampled data of ground-based time domain surveys. In particular, we describe a new technique for identifying flaring stars, which we have implemented in a publicly available Python module called "PyVAN." The approach uses the Differential Evolution algorithm to optimize parameters of empirically derived light curve templates for different types of stars to fit a candidate light curve. The difference of the likelihoods that these best-fit templates produced the observed data is then used to delineate targets that are well-explained by a flare template but simultaneously poorly explained by templates of common contaminants. By testing on light curves of known identity and morphology, we show that our technique is capable of recovering flaring status in $69 \%$ of all light curves containing a flare event above thresholds drawn to include $<1 \%$ of any contaminant population. By applying to Palomar Transient Factory data, we show consistency with prior samples of flaring stars, and identify a small selection of candidate flaring G-type stars for possible follow-up.
\end{abstract}

Unified Astronomy Thesaurus concepts: Variable stars (1761); Eruptive variable stars (476); Astronomy software (1855); Flare stars (540)

\section{Introduction}

The arrival of large-scale recent, current, and near-future ground- and space-based time domain photometric surveys (All-Sky Automated Survey for SuperNovae (ASAS-SN; Shappee et al. 2014); Dark Energy Survey (DES; Dark Energy Survey Collaboration et al. 2016), Evryscope (Law et al. 2015); Large Synoptic Survey Telescope (LSST; Ivezić et al. 2019); MEarth (Nutzman \& Charbonneau 2008); Palomar Transient Factory (PTF; Law et al. 2009); Sloan Digital Sky Survey (SDSS) Stripe 82 (Abazajian et al. 2009); Zwicky Transient Facility (ZTF; Bellm et al. 2019); Kepler (Borucki et al. 2010); and Transiting Exoplanet Survey Satellite (TESS; Ricker et al. 2015) is helping to transform many areas of stellar astrophysics. However, each of these surveys is driven by different primary science motivations, leading each to differ in their filter combinations, cadences, durations, photometric precision, and dynamic ranges. The challenge for many domains of stellar astrophysics research is how to utilize the incredible wealth of data provided by these heterogeneous surveys.

Stellar flares are produced by magnetic reconnection events in stellar photospheres that release copious amounts of energy across X-ray, UV, optical, IR, and radio wavelength regimes over timescales of seconds to days. Diagnosing the underlying stellar physics that drives these flares has historically relied on targeted observing campaigns on small numbers of individual objects (e.g., Moffett 1974; Lacy et al. 1976; Hawley \& Pettersen 1991; Kowalski et al. 2010, 2013, 2019; Fuhrmeister et al. 2011; Hilton 2011; Hawley et al. 2014; Silverberg et al. 2016; Loyd et al. 2018a), where the cadence, wavelength coverage, and/or duration of observations were optimized to constrain the detailed behavior of flares during their rise and decay phases. Large-scale time domain surveys offer the promise of studying different niches of flare physics, based on each survey's design. Analysis of larger populations of flare stars using time domain surveys that utilize sparse, long duration observations at one or more filters (e.g., Kowalski et al. 2009; Walkowicz et al. 2011; Yang et al. 2017; Schmidt et al. 2019) lack sufficient cadence and wavelength coverage to diagnose the detailed behavior of rise and decay events, but are ideal for sampling the frequency of the highest-energy, most infrequent events. Determining the frequency of these highenergy flares is of particular interest for their potential impact on the habitability of exoplanets (Segura et al. 2010; Howard et al. 2018; Loyd et al. 2018b). Analysis of large populations of high-cadence, long-duration observations in a single filter (Davenport 2016) can better diagnose the ensemble frequency of moderate-amplitude flares, including their rise and decayphase behavior, at the expense of lacking broad wavelength coverage to sample how the time-dependent behavior of the full stellar atmosphere to flare events.

Identifying and classifying the myriad of different variable sources detected in large-volume time domain surveys is a subject of critical importance. The Arizona-NOAO Temporal Analysis and Response to Events System (ANTARES), for example, is currently developing and testing machine-learning toolsets to characterize and classify variable and transient sources for LSST, to help populate data alert systems (Narayan et al. 2018). Zinn et al. (2017) have explored use of quasiperiodic oscillation stochastic process models (QPO) and damped random walk models (DRW) to explore the best methods by which to detect periodic, quasiperiodic, and stochastic variables in the OGLE survey. Because stellar flares are expected to contribute a significant number of "contaminant" transient events in large-volume surveys like LSST (Kowalski et al. 2009), determining how to identify and classify stellar flares in such surveys has a broad impact beyond the stellar flare community. Despite this, they are often not targeted for identification in the aforementioned alert systems.

Historically, studies of flares in single- to multiple-object time series data have used simple automated routines to 
identify flares in these data as events in which two or more consecutive observations exceed the background by $N-\sigma$, and sometimes relied on subsequent visual inspection of these events to verify accuracy (e.g., Davenport et al. 2014; Silverberg et al. 2016). Larger-volume surveys comprised of high-quality, quasi-uniformly sampled space-based data have explored basic flare template cross-correlation matching to detrended data (Davenport 2016) and machine-learning techniques (Vida \& Roettenbacher 2018) in order to better automate flare detections in large data sets; however, Vida \& Roettenbacher (2018) have noted some contamination issues with some of these approaches. Kowalski et al. (2009) was able to identify flare events in sparsely sampled SDSS observations of Stripe- 82 by taking advantage of the multicolored nature of these drift-scan data. The best method to identify flare events in current and future irregularly sampled, single- or multiple-filter, ground-based all-sky surveys has not been addressed.

In this paper, we explore different methods for identifying flare events from irregularly sampled ground-based time domain photometric survey data, focusing on template optimization using differential evolution. The results of the implementation of such a technique are presented here, along with PyVAN: the publicly available software created to carry it out. $^{5}$ We discuss the problems facing flare searches in irregularly sampled data (Section 4), and investigate common sources of contamination for prior automated flare searches (Section 5). We then describe the technique that our software uses to identify flaring stars while also avoiding the most common sources of false positives in flare studies (Section 6). This technique is then tested and calibrated by application to light curves of simulated PTF quality and known identity, generated by reducing Kepler short-cadence light curves to PTF-like precision and sampling (Section 7). The recovery rate of the flaring status of stars in data like PTF's is probed by application of the PyVAN software to the PTF data of prior well-studied flare samples (Section 8). Following this, we apply this technique to a selection of northern hemisphere PTF targets observable by TESS, producing a sample of strong candidates for TESS short-cadence follow-up (Section 8.3). Finally, we briefly discuss application of the software to data of other recent or upcoming surveys (Section 9).

\section{Data}

The primary data analyzed in this study were collected at the Palomar Observatory over a period of approximately seven years as part of the PTF survey (Law et al. 2009). PTF observations are taken in either SDSS $g^{\prime}$-band or Mould $R$ band, with the majority of data being collected at an exposure time of $60 \mathrm{~s}$. Additionally, PTF data was complemented with data from the Pan-STARRS1 (PS1; Magnier et al. 2013) survey taken in the $g, r, i, z$, and $y$ broadband filters to allow for color selection of the PTF targets.

\section{Target Selection}

To search for flare candidates in PTF data, the Large Survey Database (LSD; Juric 2012) is used to acquire PTF photometry meeting certain criteria. Because many PTF targets are observed in only a single filter, LSD is also used to crossmatch any potential targets with Pan-STARRS-1 (PS1) data to

\footnotetext{
https://github.com/kdlawson/pyvan
}

allow for color-color selection. For analysis of SDSS Stripe 82 flares from Kowalski et al. (2009) and Kepler flares from Davenport (2016), PS1 $g-r$ and $r-i$ colors for targets are transformed to SDSS $r-i$ and $i-z$ colors using quadratic color transformations provided in Tonry et al. (2012), which are then dereddened using the Bayestar-17 3D dust map (Green et al. 2018) in order to attain a minimum extinction value for initial selection. Unitless extinction values from Bayestar17 are converted to $g-r$ and $r-i$ corrections using coefficients provided in Green et al. (2018). Following this, we implement the color-selection criteria for M-type stars utilized by Kowalski et al. (2009). For TESS follow-up candidates, PS1 photometry is instead utilized to select targets bright enough for observation with TESS, requiring $I_{C}<13$ (Ricker et al. 2015). For every sample, PTF observations in the database are rejected if flags indicate bad astrometry, bad photometry, or the presence of halos or ghost pixels in the exposure. To avoid selection of light curves that lack statistically significant photometric enhancements, targets are required to have at least one observation that is $3+$ standard deviations brighter than the mean magnitude (a precomputed light curve statistic that can be used for selection in LSD).

Once light curves are acquired, further cuts are made as they are searched to select flare event candidates. The median absolute deviation (hereafter $\sigma_{\text {med }}$ ) is computed for each target light curve. This value is then used to select flare event candidates using a variant of the consecutive outlier test (Hawley et al. 2014). Any instance of a flux enhancement that is at least $5 \sigma_{\text {med }}$ brighter than the median value with a neighboring point at least $2.5 \sigma_{\text {med }}$ brighter is recorded as a candidate flare event to use in fitting. Targets having at least one such candidate event remain in the target pool.

\subsection{Kepler Flaring Targets}

The sample of 4041 Kepler flare star candidates from Davenport (2016) is reduced to 158 targets by selecting for only those targets that are present in both PTF and PS1 data in LSD and which meet our color selection criteria for M-type stars. From these, our database query returns a sample of light curves containing $1877 R$-band observations of 23 targets and $1860 \mathrm{~g}$-band observations of 26 targets, with 41 unique targets in total (117 are eliminated by our query cut requiring at least one bright outlier in the light curve). Following application of the consecutive outlier candidacy test, four $R$-band and six $g$-band Kepler flare candidate light curves remain for template fitting.

\subsection{SDSS S82 Flaring Targets}

Rather than starting with the 236 flaring M-dwarf stars in the Kowalski et al. (2009) sample, the entirety of the Stripe 82 region in PTF is searched for M-type candidates. The full region is selected here because both PTF and SDSS data feature irregular sampling, making the recovery of flaring targets in PTF that were not identified in SDSS likely. The initial selection of Stripe 82 targets in PTF contains 4,194,553 $R$-band observations of 37,113 targets and 1,619,796 g-band observations of 18,686 targets, with a total of 54,482 unique targets overall. Following the consecutive outlier selection process, $2804 R$-band light curves and $1934 g$-band light curves for the Stripe 82 targets remain. 
Table 1

Light Curve Metrics Inspected for Flare Star Separation in PTF

\begin{tabular}{ll}
\hline \hline Metric & Description \\
\hline$\chi^{2}$ & Chi-square metric for observations in the light curve \\
rms & Root mean square deviation for the target's magnitudes \\
Con & Fraction of light curve observations in which three consecutive observations are at least twice the rms from the median magnitude, plus one \\
Max Slope & Maximum light curve pairwise slope across all consecutive observations (Richards et al. 2011) \\
Stetson J & A measure of the degree of autocorrelation among the light curve magnitudes (Stetson 1996) \\
Stetson K & A robust measure of the kurtosis for the distribution of magnitude measurements (Stetson 1996) \\
Peak Significance & The difference between the median and minimum magnitudes, divided by the target's rms \\
Amplitude & The difference between the target's maximum and minimum magnitudes \\
Modified m-statistic & The difference between the median and minimum magnitudes, divided by the target's amplitude; modified from Kinemuchi et al. (2006) \\
$A_{95-5}$ & As amplitude, but using the 95th and 5th magnitude percentiles instead of max and min \\
\hline
\end{tabular}

\subsection{TESS Follow-up Candidates}

For the sample of TESS follow-up candidates, LSD is used to select for any Northern hemisphere targets meeting the aforementioned criteria and observable with TESS. Following the initial query, the selection includes 6,354,757 $R$-band observations of 174,707 targets and 8,315,543 $g$-band observations of 157,682 targets. After eliminating targets that fail the consecutive outlier test, the final sample includes 3234 $R$-band light curves and $2163 \mathrm{~g}$-band light curves.

\section{Identifying Flare Events in PTF Data}

Early in this investigation, attempts were made at identifying likely flare stars by use of precomputed statistics for each PTF light curve contained within LSD. In total, two-dimensional scatter plots comparing 10 statistics were visually inspected to search for promising separations of flaring stars from the rest of the stellar population and from other varieties of variable stars. The examined light curve metrics are described in Table 1. Ultimately, a number of statistics that were identified tended to differentiate some members of known variable star populations from the general population (Stetson $\mathrm{J}$ in particular). However, none of the tested relationships reliably separated known flaring stars from other types of variable stars. A substantial roadblock for this approach was the difficulty of finding a sample of target light curves in PTF in which verifiable flare events occur. While previously identified flaring stars can be selected in PTF, there are a number of confounding issues that remain:

1. A star with flares observed in some other survey may not have coverage of any flare events in PTF's data, and will thus manifest more similarly to an inactive star in light curve statistics.

2. An apparent bright enhancement in the PTF data of a known flare star may still be produced erroneously by PTF's often large photometric errors or by issues with PTF's automated photometry. Without contemporaneous observations of the target star by another survey, these possibilities are not easily distinguishable.

3. Blindly adopting prior samples risks propagation of contamination. Training new techniques with prior samples that may contain contamination can result in a new technique that is specifically engineered to accept the false positives of its predecessor.

These issues make the testing of a technique for flare finding in data like PTF's especially difficult, and they introduce a number of necessary design constraints for any such approach.
Namely, the method must be able to recover flares while rejecting flare-like events being contributed both by photometric noise and common astrophysical contaminants (see Section 5). Further, this should be achieved without training such a technique under the assumption that flare-like features in PTF light curves of previously identified flaring stars are truly flares.

\section{Flare Catalog Contamination}

As a next step, we look to prior automated flare searches to gauge what types of astrophysical variables, if any, appear frequently as contaminants. To do this, we examined the sample of flare stars from the Kepler flare catalog (Davenport 2016), perhaps the largest catalog of flare stars currently available. First, we checked the sample for overlap with the list of eclipsing binaries from the Kepler eclipsing binary catalog (Kirk et al. 2016), as well as overlap with a list of known RR Lyrae stars in Kepler (Nemec et al. 2013). Additionally, we conducted a literature review to identify variable stars of other classifications present in Kepler, including: Delta Cepheid, W Virginis, Delta Scuti, Mira, RV Tauri, Beta Cepheid, Gamma Doradus, $\alpha^{2} \mathrm{CVn}$, and ellipsoidal variables (Debosscher et al. 2011). We found no overlap between the flare sample and the identified $\alpha^{2} \mathrm{CVn}$, Mira, RV Tauri, and W Virginis variables. Each of the other groups had at least some overlapping membership with the Kepler Flare catalog. The largest contributors of potential contaminants were found to be eclipsing binaries (640 targets), Delta Scuti stars (284 targets), Gamma Doradus stars (135 targets), and RR Lyrae stars (40 targets). With 4041 targets listed in the Kepler flare catalog, these four types make up $27 \%$ of the full sample.

Of course, it is possible that the nonflaring identity (rather than the flaring identity) is erroneous or that some of these targets do truly exhibit flares. However, the appearance of nearly every RR Lyrae from the comparison list (all but the eponymous RR Lyrae itself) and the lack of mechanisms understood to produce flares in objects of this type suggest a systematic misidentification. Moreover, this process was intended primarily to identify those targets for which automated flare searches were most likely to return false positives, but it is not intended to be rigorous in terms of precise numbers of contaminants of each type. Toward the goal of identifying reliable samples of flare stars, consideration of these frequent sources of contamination is necessary in both the design and testing of detection techniques. 


\section{The PyVAN Software}

PyVAN (Python Variable Assessment with Nonlinear Template Optimization) is a parallelized, user-friendly, and publicly available software package for Python, created to aid in identifying flare star candidates in light curves having irregular or sparse sampling and sizable photometric errors. PyVAN uses the differential evolution technique (Storn \& Price 1997) to optimize fits of empirical light curve templates to the data. For each template's determined best fit, the likelihood that it would produce the observed data is computed and comparison metrics are generated. These metrics can then be used to separate flare star candidates from a sample population. The software is intended to aid in identification of flare stars by reducing large samples of light curves down to a quantity that can be more easily verified by inspection. The core components of PyVAN carry out the following tasks, as well as the initial identification of flare event candidates described in Section 3.

\subsection{Template Optimization with Differential Evolution}

LMfit is a nonlinear optimization and curve fitting package for Python that implements variables as parameter objects, and it offers a large number of prebuilt optimization routines (Newville et al. 2016). Using LMfit's parameter objects allows for easily specifying and altering bounds for each of the varying template parameters, ensuring that any returned fits are not only a statistical best fit, but also physically reasonable.

While LMfit was built to expand on the LevenbergMarquardt least-squares optimization algorithm from SciPy, early testing in this application revealed that least squares would explore only a small portion of each allowed parameter space, becoming trapped in local optimization minima. Instead, fitting is carried out using differential evolution. Differential evolution is an evolutionary optimization algorithm that, compared to least-squares optimization, requires more function calls but better explores the global parameter space. The typical implementation of differential evolution works by randomly initializing a trial solution population, "evolving" each generation by adding a weighted difference of two candidates to an additional candidate, and then accepting that new candidate if it is a better solution (Storn \& Price 1997). Each member of each generation's trial solution population also has some chance of being "mutated" to a different region of the parameter space, allowing population members to explore parameter values that might otherwise remain untouched. In this application, differential evolution allows for the best-fit parameters for each template to be identified relatively quickly, despite parameter spaces that are often quite large as a result of sparse data that offers only minimal constraints. Additionally, it eliminates the burden of determining reasonable parameter initializations, instead requiring only bounds for each. For each template's optimization, we weight the light curve's observations with the inverse of the photometric uncertainty.

\subsubsection{Flare Template}

The utilized flare template was empirically derived from Kepler observations of over 6100 flares (Davenport et al. 2014) (see Figure 1). This shape is expected to fit reasonably well to the majority of flare events, and is typically referred to as the "FRED" model, for "Fast Rise, Exponential Decay." For ease of application to PTF light curves, which are acquired in the form of magnitudes rather than fluxes, the software takes parameters in terms of magnitudes and converts these to relative fluxes internally. A target having a single candidate flare event is optimized in LMfit over a set of four parameters: quiescent magnitude, peak amplitude, flare start time, and rise duration ( $m_{0}, d m, t_{0}$, and $d t$ respectively). In the adopted model, the ratio of rise duration to decay duration (and thus total duration) is fixed. Given that rise duration is likely never measurable with any meaningful precision in PTF data, this parameter is more accurately a proxy for the flare decay duration or total duration. For studies analyzing flare photometry with very high cadence, in which rise duration can be measured independently of decay duration, the PyVAN software can be altered trivially to fit for these durations separately. For the purpose of analyzing PTF data, however, adopting the template as presented is sufficient. Boundaries for the utilized parameters are set both for logical limitations (i.e., no negative durations or amplitudes) and for approximate observed limitations (to ensure that determined flare fits have plausible parameters).

For targets having multiple candidate flare events, the approach is somewhat more complicated. The candidate events are first sorted by their apparent amplitudes (descending). Then, up to three flare events are fit simultaneously, optimizing for a single quiescent magnitude as well as a start time, rise duration, and peak amplitude for each. By fitting multiple candidate events together, a single event is prevented from unduly influencing the placement of the quiescent magnitude, which could result in a value that poorly explains the remaining events. While fitting all candidate events simultaneously might produce a better result by ensuring that the quiescent magnitude which best explains the entire set of candidate events is found, doing so results in massively increased computation time for targets with large $N_{\text {flares }}$ in exchange for a negligible improvement (if any) in the resulting solution. In testing, fitting up to three candidates together was found to produce models indistinguishable from those resulting from larger numbers of simultaneous events for a more manageable increase in computation time. The solution found here is then used as the initial model for any remaining flare candidates, which are successively fit using only three parameters (sans quiescent magnitude) and appended to the model. Throughout this process, any flare candidates not already fit or currently being fit are masked to prevent them from affecting the results of other fits. When completed, the solution has $3 N_{\text {flares }}+1$ parameters, where $N_{\text {flares }}$ is the total number of candidate flare events in the light curve. The number of peaks fit simultaneously can be altered using a keyword argument in the software. In many cases, the need for this procedure might be circumvented by adopting some version of a clipped average as the quiescent magnitude, thus eliminating $m_{0}$ from the set of optimization parameters. However, when sparse sampling results in a light curve with an especially well-sampled flare and poorly sampled quiescence, the implemented procedure achieves better performance without compromising general efficacy.

\subsubsection{Quiet Template}

To test the likelihood that an observed light curve was produced by photometric errors alone, a simple flat line is also fitted to each candidate, seeking the $m_{0}$ that optimizes the fit. For this purpose, least-squares optimization is sufficient, as the 


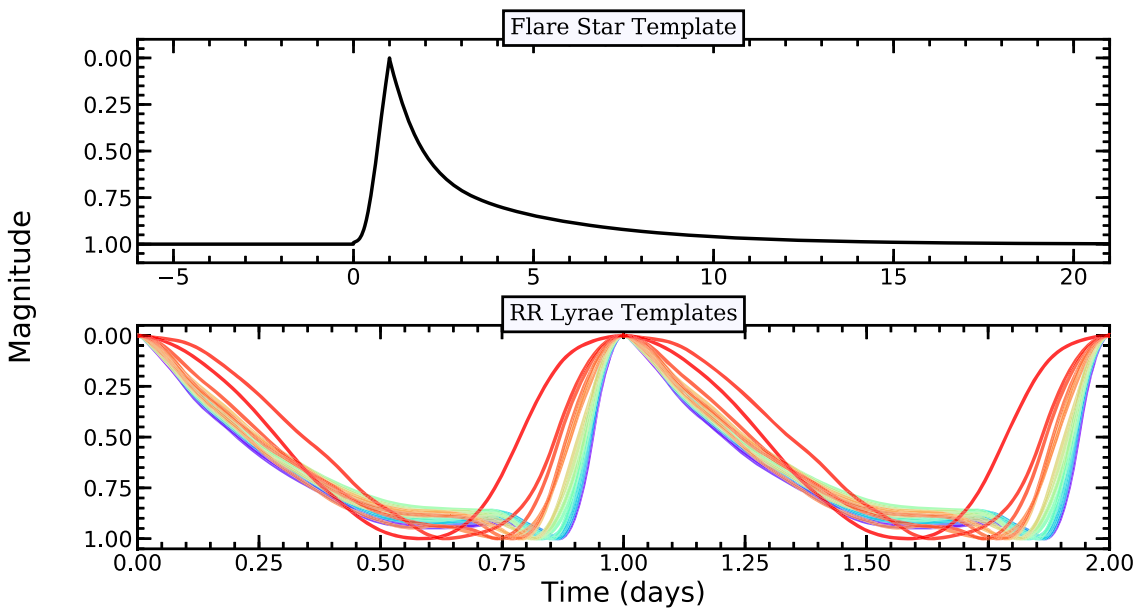

Figure 1. Top: empirically derived flare template from Davenport et al. (2014), parameterized in terms of quiescent magnitude, peak amplitude, flare start time, and rise duration $\left(m_{0}, d m, t_{0}\right.$, and $d t$ respectively). As depicted, the template has values of $m_{0}=1, d m=1, t_{0}=0$, and $d t=1$. Bottom: two periods of empirically derived $g$-band RR Lyrae templates from Sesar et al. (2010), parameterized in terms of base magnitude, amplitude, phase offset, and period ( $m_{0}, d m, t_{0}$, and $d t$ respectively). Templates depicted have values of $m_{0}=1, d m=1, t_{0}=0$, and $d t=1$. Notably, both the flare template and RR Lyrae templates feature tall, sharp peaks; this, combined with sparsely sampled data, can result in light curves whose identities are difficult to distinguish.

optimization's sole minimum will be the global minimum. For light curves of many observations, such as those of Kepler, one would expect that the quiescent magnitude found in flare fitting and the one found here would be very nearly identical. However, with light curves like those of PTF, they can diverge considerably. This occurs most radically for light curves in which a small number of points are dominated by a single large-amplitude flare event or when observations of a flare event exhibit much smaller photometric errors.

\subsubsection{RR Lyrae Templates}

RR Lyrae variables are periodically pulsating variable stars with particularly sharp light curve features having large amplitudes (Sesar et al. 2010). For this reason, contamination from RR Lyrae stars is of particular concern for flare searches. While more continuous data may allow for these targets to be filtered by applying techniques such as period folding, the poor time coverage of data like PTF's makes such detections uncertain. Moreover, the light curves of flaring stars often exhibit periodic variability as a result of starspots, and may therefore result in strong responses in periodograms (with periods similar to those of RR Lyrae or other common contaminating variables). Using differential evolution to fit sets of empirically derived RR Lyrae templates to each light curve, the possibility that a flare star candidate is an RR Lyrae star instead can be evaluated.

We utilized $23 g$-band and $22 r$-band templates that described the majority of RR Lyrae analyzed in Sesar et al. (2010). Each filter set includes two templates for RRc targets, with the rest being for RRab targets. Though created from SDSS observations, the chosen templates are found to fit RR Lyrae in PTF sufficiently for our purposes. The templates are acquired in the form of time and magnitude coordinates covering one period of the RR Lyrae variation. These are implemented in PyVAN by interpolating over the provided values to create functions that can be passed a set of observation times and RR Lyrae parameters, and which return a corresponding set of magnitudes. The four parameters-base magnitude, amplitude, phase offset, period $\left(m_{0}, d m, t_{0}\right.$, and $d t$ respectively) — are again allowed to vary over ranges loosely constrained by observed RR Lyraes.

For optimization, differential evolution alone proved to be time-consuming and often resulted in poor fits, with results changing meaningfully from run to run and varying more than expected between similar templates (as a result of differential evolution's metaheuristic and stochastic nature). Instead, a hybrid optimization procedure is implemented, utilizing the fact that the shape of each subsequent template changes only slightly from its predecessor (see Figure 1). The first template is fit repeatedly using differential evolution (12 times by default), saving the results of the best fit from among them. Repeated fitting this way was found to produce superior results and to do so with shorter computation times compared to altering the available optimization parameters. The first template's best fit is then used to initialize the second template's fit, which then uses least-squares optimization instead of differential evolution. The effectiveness of least squares here depends on the fact that the global minimum in the parameter space will have moved only slightly for the new template, and that the local minimum that least squares finds will actually be the global minimum for the new template. Following this, each template is initialized with the best fit parameters of the previous template, and optimized with least squares. In testing, this process not only produces fits superior to those of the individual constituent routines (see Figure 2), but also requires much less time to employ than differential evolution alone. With typical photometric uncertainties, the final template choice may improve fit metrics only slightly (such that any one template can usually allow an RR Lyrae star to be distinguished). However, the processing time is dominated by the differential evolution portion such that fitting the first template takes many times longer than fitting all of the remaining templates using leastsquares optimization.

\subsection{Comparison Metrics}

For each target, the best parameters for all three fit templates are stored, as well as a log-likelihood for each of these fits. We compute log-likelihood as a function of the $\chi^{2}$ of the residuals between the observations and the best-fit model as well as the 


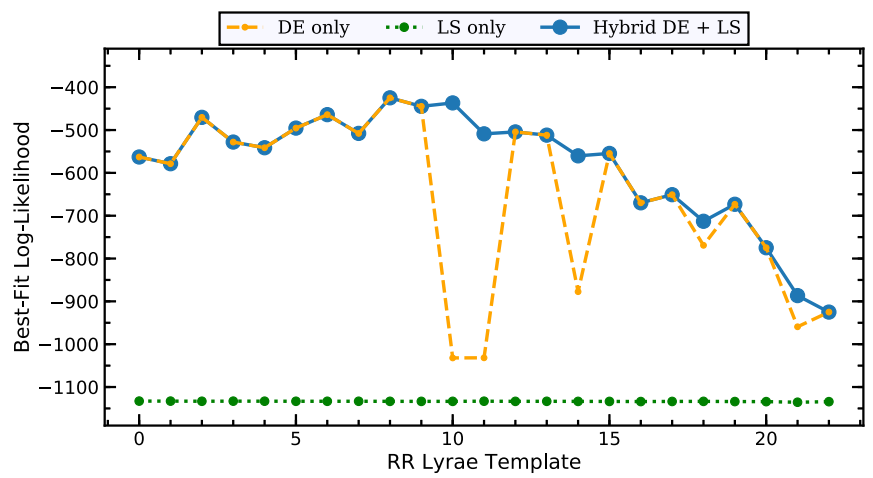

Figure 2. A comparison of best-fit log-likelihoods for three different optimization techniques (see Section 6.1.3) used to fit 23 RR Lyrae templates to the light curve of a known RR Lyrae star in PTF. Pure least squares (green dotted line) provides a consistently poor fit, becoming trapped in local minima and exploring very little of the parameter space. Pure differential evolution (gold dashed line) generally provides good fits, but is time-consuming and sometimes converges to a suboptimal solution (for at least five templates here). The hybrid technique (blue solid line) fits the first template repeatedly using DE and then initializes each subsequent template for optimization with least squares using the best-fit parameters of its predecessor. This results in a more consistently strong fit and takes less time than a pure DE optimization.

number of observations in the light curve:

$$
\begin{aligned}
& \ln (L)=\ell=-\frac{n_{\mathrm{obs}}}{2} \cdot \ln \left(\frac{\chi^{2}}{n_{\mathrm{obs}}}\right), \\
& \chi^{2}=\sum_{i=1}^{n_{\mathrm{obs}}} \frac{\left(m_{\mathrm{obs}, i}-m_{\mathrm{model}, i}\right)^{2}}{\sigma_{i}^{2}}
\end{aligned} .
$$

To identify strong flare candidates and eliminate likely false positives, the differences of best-fit log-likelihoods between each target's templates are computed. These include: (flarequiet), (flare-RR Lyrae), and (RR Lyrae-quiet); henceforth $\Delta \ell_{f q}, \Delta \ell_{f r}$, and $\Delta \ell_{r q}$, respectively. A strong flare candidate is expected to demonstrate meaningfully positive values in both $\Delta \ell_{f q}$ and $\Delta \ell_{f r}$. Though a true flaring star with large errors or unfortunate sampling may manifest with low values in these metrics, such a target is considered to be "unrecoverable" in that the observed target's identity cannot be confidently determined with the available information.

A candidate's $\Delta \ell_{f q}$ value is an indicator of the statistical significance of its flare event candidate(s); a large $\Delta \ell_{f q}$ indicates a small chance that the observed features could be produced purely by the recorded photometric error. Because the flare model consists of a quiescent model with one or more deviations to include outlier flare candidates, $\Delta \ell_{f q}$ should never manifest as significantly negative.

Meanwhile, $\Delta \ell_{f r}$ serves primarily as an indicator of the ambiguity of the candidate light curve's shape with respect to the adopted flare model. A significantly negative $\Delta \ell_{f r}$ indicates that the candidate's light curve morphology is better explained by some aspect of an RR Lyrae template. A small value of $\Delta \ell_{f r}$ may indicate that the observed candidate event is perfectly consistent with the flare template, but that the information available is insufficient to rule out shapes consistent with the light curve morphologies of RR Lyrae stars.

We do not utilize $\Delta \ell_{r q}$ for flare identification. While it would likely be helpful in searching irregularly sampled data for RR Lyrae (in place of $\Delta \ell_{f q}$ ), three log-likelihood values allow for only two nondegenerate $\Delta \ell$ values (i.e., it provides no unique information for identifying flaring stars).

\subsection{Processing Times}

PyVAN carries out fitting a target for all desired templates on a single processor core, distributing candidate light curves in a sample to multiple cores for fitting as available. A typical PTF light curve with a single flare candidate is fit with a flare template and a quiet template in $\sim 0.6 \mathrm{~s}$ on a modern consumer processor. Meanwhile, fitting the set of RR Lyrae templates takes closer to $10 \mathrm{~s}$ per target. The long fitting time for RR Lyrae templates can be mitigated in practice by applying these only to targets demonstrating a promising $\Delta \ell_{f q}$ following flare and quiet fitting (as described in Section 8). On average, over all of the samples discussed in Section 8, this procedure allowed processing of around 0.3 targets per second per core for PTF light curves. This works out to around 21 hours on a four-core system, to process a large sample of $10^{5}$ PTF targets having candidate flare events.

\subsection{Software Features}

PyVAN is designed to be easily altered and appended, and delivers products as Python dictionaries. The software includes functions to carry out the core of the PyVAN data reduction process, including fitting of flare, quiescent, and RR Lyrae templates, as well as additional functions for plotting result metrics and displaying the template fits for individual targets.

In addition to carrying out the core features described above, PyVAN also implements a number of additional features, including many for visualizing fitting results. Users can easily generate scatter plots of computed $\Delta \ell$ metrics, as well as displaying an interactive light curve for an individual target of interest (which can be selected based on a target's location in the scatter plots), with buttons to overlay any of the fit template solutions. PyVAN also includes functions to aid in the generation of test data (as in Section 7), in which a set of high-quality light curves or light curve models can be used to simulate data of similar quality to an input lower-quality data set.

Further, PyVAN allows users to input additional templates to fit to data, allowing functionality for groups particularly concerned with other contaminants or interested in identifying targets other than flare stars. New templates can be implemented in one of two ways. The first method is to use a generalized procedure to optimize a desired template, in which the user provides a set of parameters, parameter bounds, and a Python function for the template (which takes arguments of a time array and the template parameter values and returns a corresponding array of magnitudes). The second option allows a user to create a more nuanced template-fitting procedure as a Python function (such as those implemented for our flare and RR Lyrae templates), which takes an array of data containing (at least) observation times, magnitudes, and errors, and returns a Python dictionary containing fit parameters and a loglikelihood value for the best fit to the data.

\section{Testing and Calibrating Using Data of Simulated PTF-quality}

When analyzing poorly sampled data of a target of known identity, there is no guarantee that any archetypal features will be discernible or real. To conduct a meaningful examination of PyVAN's techniques in application to data like PTF's, it is therefore helpful to simulate PTF quality in data for which the target's identity is known and where the underlying light curve 
shape is much better constrained. This a priori knowledge can then be combined with the metrics that result from our procedure to gauge the effectiveness of the approach and to inform selection of targets based on these metrics in later application to real data.

\subsection{Creating Data of Simulated PTF Quality}

To begin this process, we utilize a selection of short-cadence Kepler light curves of targets indicated as flare star candidates in Davenport (2016), including both true flare stars and a number of apparent contaminants (identified in Section 5). These serve as the "donor" light curves from which we will produce light curves of PTF quality. The pool of contaminants includes RR Lyrae, eclipsing binary, Delta Scuti, and Gamma Doradus stars, as these groups represent the most frequent contaminants to the Kepler catalog in terms of raw number. For each donor light curve, a central flux value is computed by clipping any values further than $2 \sigma_{\text {med }}$ from the median and computing the error-weighted average of the remaining values. This central flux is then subtracted from each light curve, and a simple linear detrending process is performed to eliminate large systematic effects between quarters. All quarters of shortcadence data are then combined into a single continuous light curve and converted to relative magnitudes. Following this, each composite light curve is split into separate light curves anywhere that more than five days have elapsed without observations (typically where a full quarter or more of data is missing). Separating the light curves this way results in approximately continuous data that can be more accurately convolved with PTF's sampling without eliminating observations. These light curves are then placed into a pool corresponding to the target's suspected identity. Once each pool is created, we visually inspect every light curve to verify the suspected identity, check that data from separate quarters are well-aligned, and ensure that no true flare events appear in any of the contaminant pools.

Next, the initial sample of PTF observations described in Section 3 (i.e., prior to elimination of targets not meeting our consecutive outlier requirement) is used to prepare pools of observing intervals, quiet magnitudes, and photometric uncertainties for simulating PTF quality data. For each light curve, both the time between each successive observation and the $\sigma_{\text {med }}$ clipped quiet magnitude is calculated and stored. Magnitude uncertainties are prepared from the full set of observations by creating magnitude bins of width 0.02 at increments of 0.01 (such that they overlap) over the span of observations and storing the corresponding uncertainties. This allows a subpool of representative magnitude uncertainties to be attained for any given magnitude while also preserving the variance of PTF's uncertainties.

To simulate a PTF-quality candidate light curve using the pools described above, we implement the following procedure. For the type of target being simulated, a prepared light curve is drawn from the respective pool of donor light curves from Kepler and the time of its first and last observation is noted $\left(t_{0}\right.$ and $t_{f}$ respectively). Next, we draw a single quiet magnitude value and add it to the selected Kepler light curves magnitudes. From the pool of PTF observation intervals, a random value is drawn, " $d t$," and the first simulated data point is placed at time $t_{1}=t_{0}+d t_{1}$. Successive values are drawn and added to the previous points time to create additional data points, ceasing at time $t_{i}$ when $t_{i}+d t_{i+1}>t_{f}$. Each simulated observation time is matched to an observation in the input Kepler light curve, allowing the simulation observation times to be perturbed from their exact drawn position by $60 \mathrm{~s}$ in either direction, to match with one of the donor observations, after which the observation times and magnitudes of the matched donor observations are stored. This allowed shift in time avoids more significant issues resulting from attempting to rebin the Kepler data, in exchange for a minor cost in terms of the accuracy of the simulation to true PTF data. Any drawn points falling more than $60 \mathrm{~s}$ from a real point in the input Kepler data are removed. Next, for every obtained magnitude value, an uncertainty (" $\sigma_{\text {PTF }}$ ") is drawn from the corresponding subpool of PTF uncertainties. Each simulated observation's final magnitude is drawn from a Gaussian distribution centered on the selected donor magnitude with standard deviation $\sigma_{\mathrm{PTF}}$. While the input Kepler data is already displaced from its "true" position by its own photometric uncertainty, the reported uncertainties for utilized PTF photometry are typically more than 200 times larger than those of Kepler photometry. As such, the inaccuracy incurred by this approach is negligible.

For each desired light curve, the process above, following the selection of an input Kepler light curve, is repeated until the resulting light curve has at least 10 observations and passes the consecutive outlier test described in Section 3. This helps to ensure that each simulated light curve will be as comparable as possible to real data being fit by the software. The number of attempts required to produce each target is tracked throughout this process and stored for use in later analysis. Hereafter, we refer to these Kepler-derived light curves of simulated PTF quality as simply "simulated light curves" or "simulated data." In total, we produce a sample of 2500 simulated light curves of each contaminant type, along with 5000 simulated light curves of flaring stars.

\subsection{Comparison to Real PTF Data}

The described process results in data that are generally comparable to real PTF data. The decision to reselect a quiet magnitude for each attempt results in distributions of quiet magnitudes and magnitude errors that appear to be organically biased toward the same types of light curves that we find in our real sample following application of the consecutive outlier test (see Figure 3 ). The most notable divergence of the simulated data results from the difference between the durations of the Kepler and PTF surveys. The utilized short-cadence Kepler data span a few hundred days at most for a given target, while PTF data frequently span over a thousand days. It may seem reasonable to circumvent this issue by simply taking a given Kepler light curve as representative of the target's behavior, and to instead duplicate Kepler light curves end-to-end in order to accommodate PTF's longer observing span. However, every type of object we are interested in testing has some degree of periodic flux modulation. At a minimum, this approach would require precisely aligning each set of observations in order to avoid disrupting this periodicity, which would otherwise weaken the periodic RR Lyrae template fit and thus delegitimize the results. More troublesome still, long-term variations in star spot modulation of flare stars or in the amplitudes and periods of RR Lyrae stars (the Blazhko effect; see Blažko (1907)) would be very difficult to account for. As such, the resulting simulated light curves must either fail in replicating the typical number of observations or the typical sampling rate of a PTF light curve. We chose to focus on best 

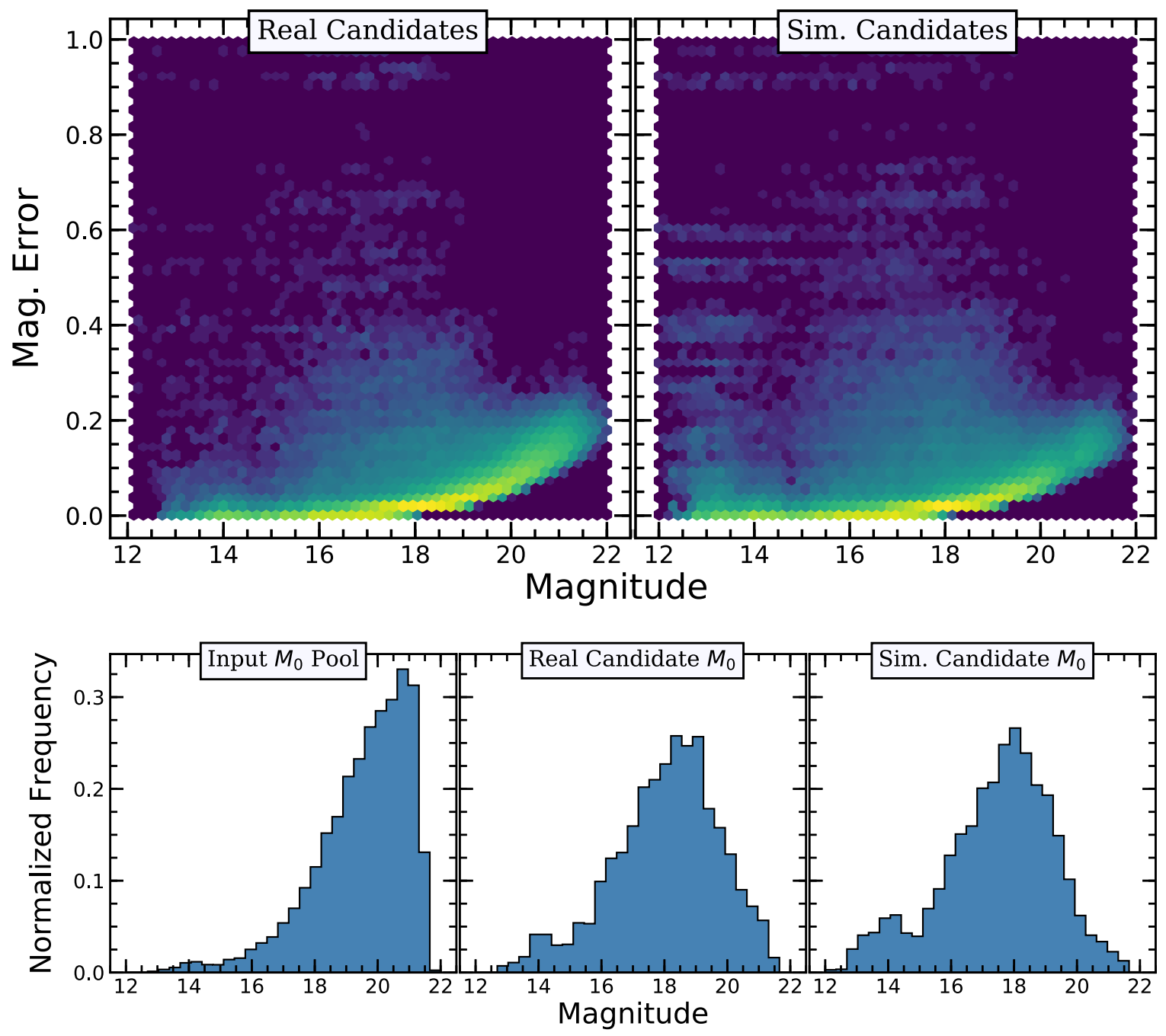

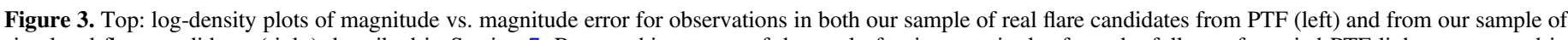

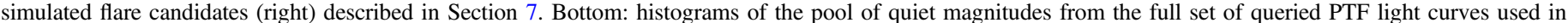

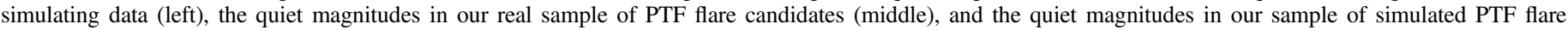

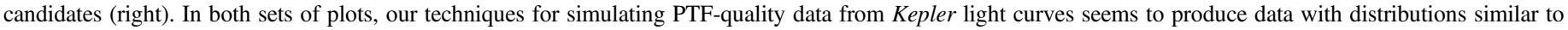
those of our real candidate sample.

reproducing PTF's sampling rate, as the alternate solution introduces the possibility of producing simulated data whose identity is easier to determine than the real data as a result of higher observation density. To mitigate the effect of this, donor light curves were chosen from among the most observed Kepler targets of each identity.

Additionally, we have made no effort to eliminate any Kepler-specific systematic effects (save for eliminating the jump discontinuities between quarters by applying a linear flux offset) or to induce any PTF-specific systematic effects in our simulated data. As the flags for both surveys are incomplete, the effects of this (if any exist) are difficult to estimate.

\subsection{Applying PyVAN to Simulated Data}

Applying the fitting algorithms and computing comparison metrics shows a meaningful correlation for simulated flare stars in the positive $\Delta \ell_{f q}$, positive $\Delta \ell_{f r}$ direction (Figure 4). An example of a strongly correlated flare fit to a simulated flare star can be seen in Figure 5.
However, there exists a sizable population of flare stars whose fit metrics place them near the origin, mixing significantly with contaminant populations. Cuts can be made in this two-dimensional space to select a sample of targets containing flares with some inclusion of contaminant populations. Before deciding a selection criteria, we consider the different factors that are likely to result in weak metrics for a simulated flare star:

1. No sampled points coincide with a real flare event (sampling failure).

2. Sampled flare points have sufficiently large uncertainties so as to make recovery above a particular selection criteria impossible (quality failure).

3. Algorithm failed to identify a strong available model fit (optimization failure).

4. FRED flare model built onto linear quiescence poorly describes observed light curve (model failure).

We take steps as follows to delineate between these cases. This process is used to gauge the effectiveness of the software and to guide decisions as to what regions of the $\Delta \ell$ space should remain in the candidate pool following application to real data. 

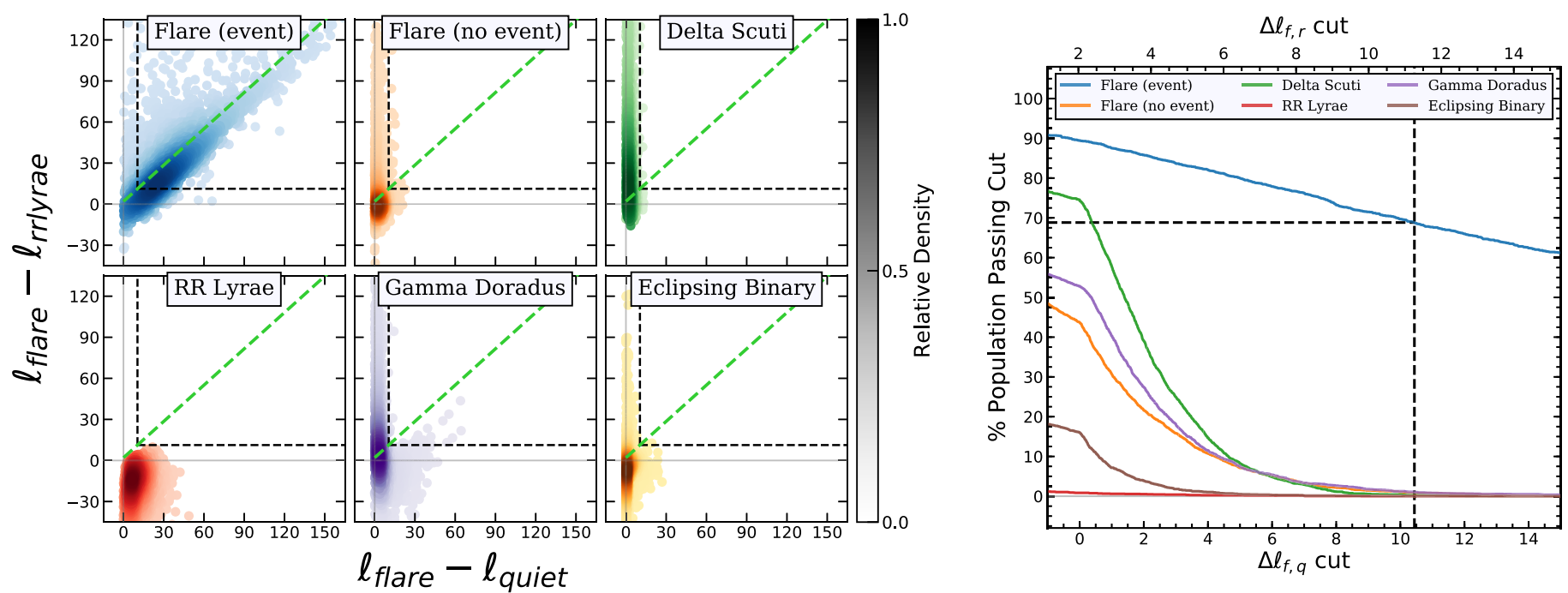

Figure 4. Left: scatter plots of $\Delta \ell_{f q}$ vs. $\Delta \ell_{f r}$ for fits to simulated PTF data, with color saturation corresponding to the relative density of the population (determined using kernel density estimation). The population of simulated flare stars is separated into two subpopulations based on whether or not their light curves have coverage of a true flare event: "flare (event)" and "flare (no event)." The dashed green line is the linear fit to the "flare (event)" population, while the dashed black lines represent the prescribed cuts on $\Delta \ell$ metrics above which $<1 \%$ of any contaminant population is found. Right: the percentage of each population included when applying a range of cuts extending from the dashed green fit line in the left plots. The $\Delta \ell_{f q}$ values of the cuts are along the lower $x$-axis, while the $\Delta \ell_{f r}$ values of the cuts are along the upper $x$-axis. The vertical dashed line corresponds to the $<1 \%$ contamination threshold depicted in the scatter plots to the left, while the intersecting horizontal dashed line gives the flaring population's throughput for that cut: $69 \%$.

\subsubsection{Sampling Failure}

By design, simulating PTF data using much higher-quality Kepler light curves allows us to determine whether or not a real flare event was included in our simulated data. We define the location of a flare in the Kepler data as any point more than $3.5 \sigma_{\text {med }}$ brighter than the median of the prepared donor light curve. This criteria is an approximation intended to identify flare features that should be recoverable. It will exclude any very small events or events embedded in star spot modulation, while also including some points which may not be feasibly recoverable with PTF's uncertainties. We then designate simulated flare targets as "flare (event)" or "flare (no event)" based on whether or not they include a detection of such an event. Henceforth, a flaring target refers to only those simulated light curves containing coverage of an actual flare event.

The resultant sample of flaring light curves includes 2101 targets, or $42 \%$ of the original 5000 simulated flare star light curves. At this point, we use linear regression to fit a line to the $\Delta \ell$ distribution for flaring stars. While outlier clipping could be used to identify a line that passes through the highest-density region of the data, allowing the line to be displaced by the asymmetric distribution to the position depicted in Figure 4 ultimately results in superior recovery rates. We then prescribe use of cuts in the positive $\Delta \ell_{f q}$ and $\Delta \ell_{f r}$ directions emanating from this best-fit line, where targets exceeding the threshold in both metrics would remain in the flare candidate pool (see Figure 4). As the position of this cut along the best fit line approaches the origin, both more flaring targets and contaminant targets will be included in the resulting sample. The ultimate position of the cuts should be determined by the number of candidates that the user is willing or able to visually inspect. For the purpose of subsequent application and analysis herein, we place these cuts such that $1 \%$ or less of any single contaminant population is included. This results in cuts requiring $\Delta \ell_{f q}>10.44$ and $\Delta \ell_{f r}>11.26$. This region contains $69 \%$ of the flare star simulations with an event, leaving $31 \%$ of these (or 13\% of the full flare star sample) as unrecovered and (thus far) unexplained.

\subsubsection{Quality Failure}

As a result of PTF's often sizable photometric uncertainties, it is possible for a sampled flare event to manifest with uncertainties large enough to preclude the possibility of recovery above the chosen cuts by making the light curve too statistically ambiguous for meaningful distinctions between templates. As with targets having no sampled flare events, we consider such a target to be unrecoverable using available information, and therefore an unmitigable loss. To identify cases where this has occurred, another metric is computed for the simulated flaring targets. In addition to the log-likelihoods for the flare, RR Lyrae, and quiet models, we add an "input" log-likelihood in which the residuals between the original Kepler magnitudes and the simulation magnitudes, weighted by the simulation uncertainties, are used to compute the metric. This value represents the measurement that would be achieved if the software exactly recovered the input light curve's original shape. The difference between the determined flare likelihood and this input likelihood, hereafter $\Delta \ell_{f}$, serves as a measure of the quality of the determined flare model. A strongly negative $\Delta \ell_{f i}$ indicates that some model exists that would produce a much higher likelihood than the one that was found, while values near zero indicate that a good fit was achieved. A flaring target that was not recovered above our cuts is deemed unrecoverable if, even with the input likelihood serving in place of the flare likelihood, the target still falls below the $\Delta \ell_{f q}$ and $\Delta \ell_{f r}$ thresholds. Applying this technique, we find 336 such unrecoverable targets among the remaining flare star simulations, leaving 319 in one of the final two categories.

\subsubsection{Optimization Failure}

It remains possible that the PyVAN algorithm has failed to identify a strong available fit for the flare model, or that the 


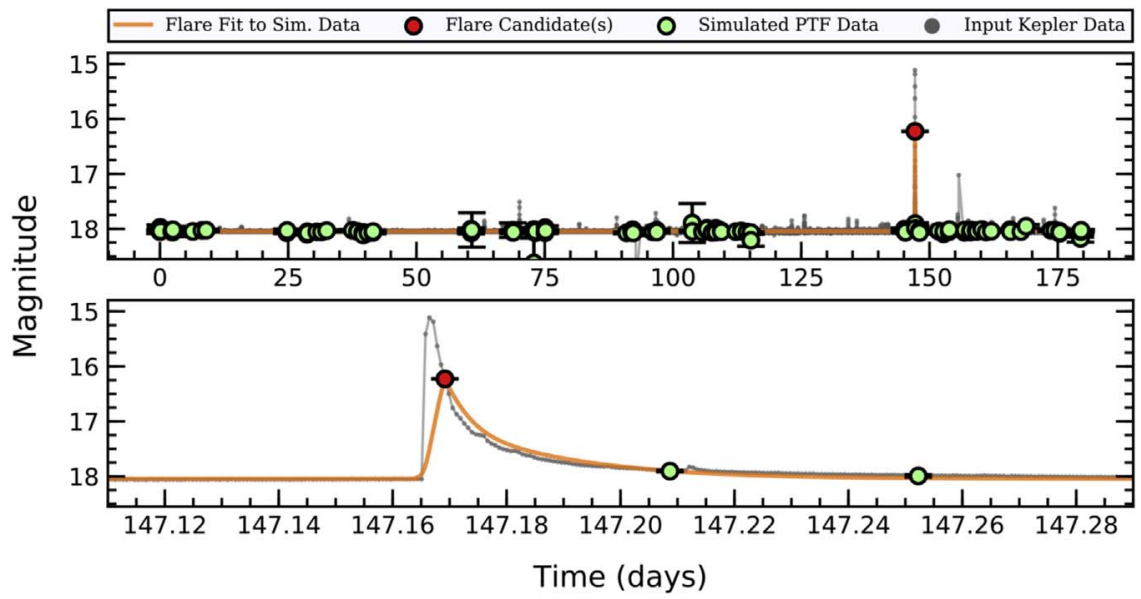

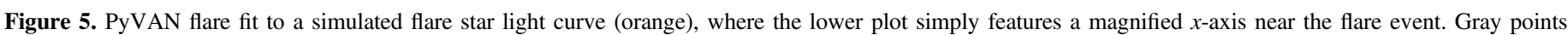

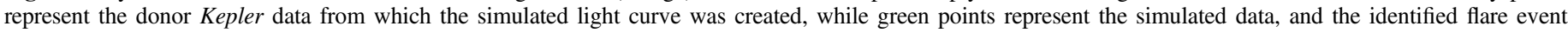

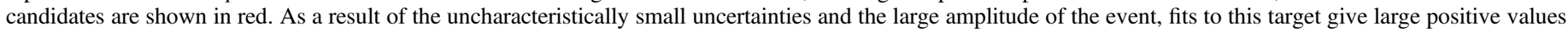
for both $\Delta \ell$ metrics.

utilized model has failed to adequately describe the flare event in the simulated light curve. The former case would result if the differential evolution procedure converged to an inadequate solution because it either failed to explore a preferable one or it was restricted from a superior solution by our parameter boundaries. To test this possibility, we open the parameter boundaries on flares to arbitrarily large or small values, increase the population of instantiated differential evolution test solutions dramatically, and then refit these targets repeatedly. Refitting each of these 319 targets 10 times results in eight targets that improve sufficiently to pass our cuts. In each one of these cases, the new solution is within the software's original parameter boundaries, indicating that the software simply converged initially to an suboptimal solution. Comparing these light curves with the set that passed without incident (1446 targets), we find that the number of observations in targets that were recovered only after refitting is $\sim 25 \%$ lower on average. Based on this, the fraction of targets for which a poor solution is found may be inflated by the smaller number of observations typical in the simulated data. To minimize this effect in general application, simply refitting any targets falling near the thresholds would be sufficient.

\subsubsection{Model Failure}

Though other factors may be to blame in isolated cases, the remaining 129 targets might be interpreted as failures of the utilized flare model to explain the observed behavior. Among other things, this could result from a flare event that has a distinctly different shape from the archetypal FRED model or from a quiescent profile that is poorly explained by a linear model due to starspot modulation (though the latter is rare, given PTF's typical photometric uncertainties). In the first case, implementation of other flare model shapes could mitigate this loss-but may also serve to erroneously pass more contaminants. For instance, implementing a template for a Gaussianshaped flare might also result in more frequently mistaking Gamma Doradus features (See Figure 7) for flare events.

The latter might be mitigated by use of a detrending routine to eliminate periodic behavior before carrying out the flare fitting routine. However, without a priori knowledge that the target is a flare star featuring significant spot modulation, it is difficult to determine when such a treatment should be applied or when it can be applied safely for PTF data. Implementing an automated detrending procedure for use on PTF data meeting some criteria is possible, but may well result in an increase of contaminants in excess of the small number of additional flaring stars recovered. Out of the 311 simulated flaring targets labeled as unrecovered due to model failure, Lomb-Scargle periodograms (Lomb 1976; Scargle 1982) identified 214 targets with periods having Lomb-Scargle powers over 0.25 , the default threshold used to warrant detrending in Davenport (2016). However, only 13 of these periods have a LombScargle power of over 0.25 in the original Kepler data, with all but 25 lying below even a power of 0.01 . This suggests that the vast majority of strong periodicities identified this way will be inaccurate. While these ratios are likely affected by the reduced number of observations in the simulated data as compared to real PTF data, applying this test to only targets having 100 or more observations still results in only $\sim 20 \%$ of detected powerful periods manifesting in the real data. Further, the longer time span of observations for real PTF light curves is more likely to be in excess of the lifetime of star spots, meaning that they cannot be assumed to manifest with stable periodicity. For general application, the failure of the chosen flare light curve model for a small number of our simulated targets is accepted in lieu of any safe alternative.

It should also be noted that some number of light curves containing potentially recoverable flare events are also lost due to our candidacy requirement for at least two consecutive outlier observations. We exclude these targets to keep our resultant sample manageable and to avoid inclusion of candidate events that cannot be easily disentangled from nonflare causes, such as cosmic rays or errors in the automated photometry procedure.

\subsubsection{Flare Star Simulations Summary}

From 5000 simulated light curves of flaring stars that pass our candidacy test, we recover flaring status in 1446 targets (29\% of the full sample) based on $1 \%$ contamination thresholds. We find that 319 targets (6\% of the full sample) could have been recovered above the same thresholds with a different model or different model parameters, while 3235 targets $(65 \%$ 

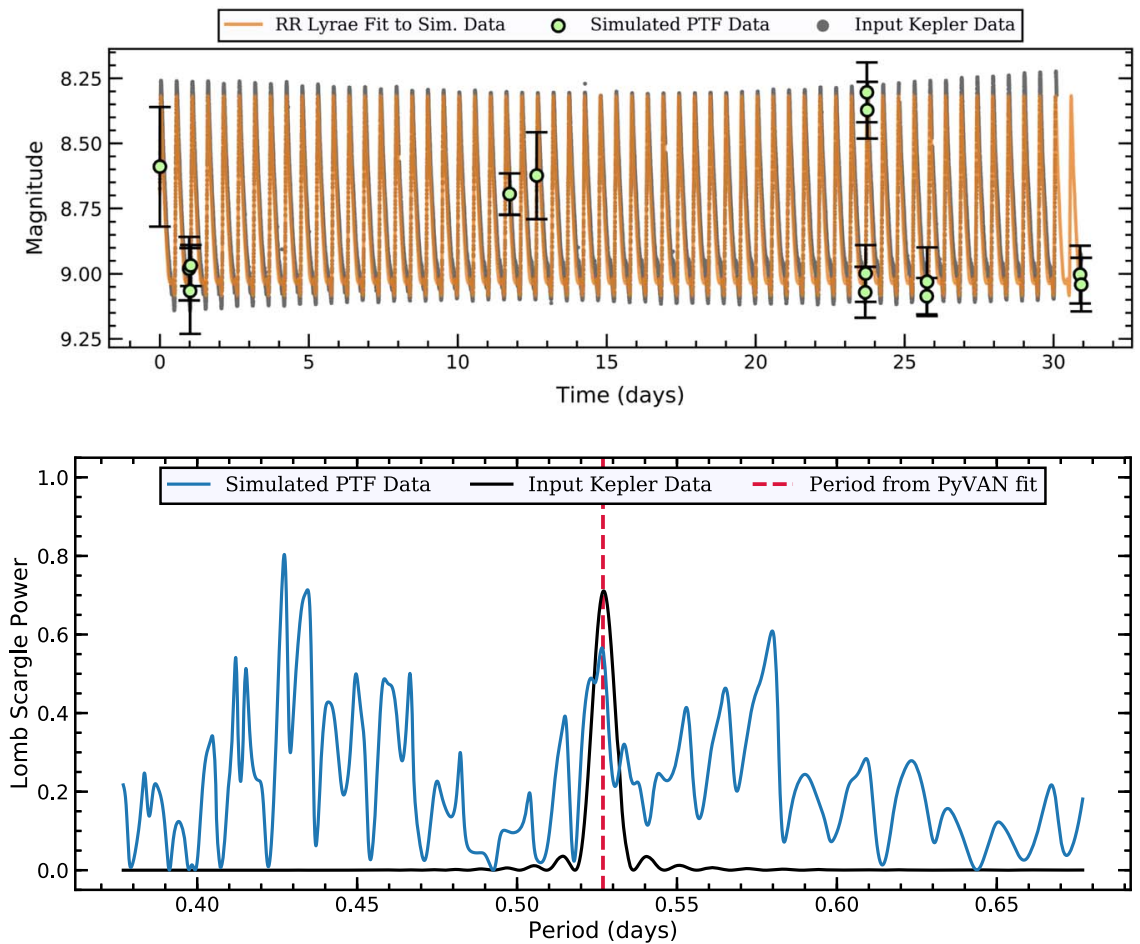

Figure 6. Top: PyVAN RR Lyrae template fit (orange line) to a simulated RR Lyrae target having only 14 observations over around 31 days. Gray points represent the donor Kepler data from which the simulated light curve was generated, while green points represent the simulated data. Bottom: Lomb-Scargle periodogram of the simulated data (blue) and the input Kepler data (black), as well as the period identified by PyVAN (red dashed line) for the simulated target above. Despite the inability of the Lomb-Scargle periodogram to detect the true period in the simulated data, the period determined by PyVAN template fitting falls within $19 \mathrm{~s}$ of the best LombScargle period for the full Kepler light curve. By making a similar comparison for other RR Lyrae simulations, our testing indicates that the accuracy of the period from PyVAN's fitting constitutes a meaningful improvement over that of the Lomb-Scargle periodogram for light curves having fewer than around 40-50 observations. This includes $\sim 20 \%$ of targets in our samples of PTF targets overlapping Kepler and SDSS S82, and $\sim 70 \%$ of targets for possible follow-up with TESS.

of the full sample) are likely unrecoverable, due to the absence of sampled flare events or large uncertainties. If we instead consider only targets found to have recoverable flare events (1765 targets), we successfully recover flaring status for $82 \%$.

Combining this information with stored information regarding the numbers of simulated flare star light curves that did not pass our initial cuts (and so were discarded), we can approximate the overall rate at which we expect to recover the identity of flaring stars in PTF data using PyVAN. We find that $5.5 \%$ of all simulation attempts resulted in a target passing our cuts. Given that we recover flaring status in $29 \%$ of all simulations passing this cut, we can estimate the overall flare recovery rate as around $1.6 \%$. We note, however, that the lower number of observations typical in our simulated data reduces both of these measurements, and so the estimated recovery rate is probably lower than would be found in application to real data.

\subsubsection{RR Lyrae Simulations}

The distribution of RR Lyrae in $2 \mathrm{D} \Delta \ell$ plots after fitting is meaningfully separated from that of the flaring population and contributes no targets as contaminants in the region above the $<1 \%$ contamination cuts. Despite the fact that the shapes of RR Lyrae in Kepler's bandpass are not perfectly described by the available RR Lyrae templates, the prescribed schema of $\Delta \ell$ cuts allows for recovery of more than $90 \%$ of all simulated flaring light curves before more than $1 \%$ of the RR Lyrae simulations are included; the implemented procedure appears to effectively eliminate RR Lyrae stars as targets of concern in data of PTF quality. An example of a PyVAN RR Lyrae fit to simulated data can be seen in Figure 6.

\subsubsection{Results for Other Simulated Targets}

Fits to simulated targets of other identities, including eclipsing binaries, Delta Scuti, and Gamma Doradus stars, tended to result in nearly Gaussian distributions in $\Delta \ell$-space, with centers lying slightly positive along $\Delta \ell_{f q}$ and slightly negative along $\Delta \ell_{f r}$. A small number of each population appears in the region dominated by flaring stars, with passing $\Delta \ell$ metrics. Typically, this seems to occur because of unfortunate sampling or large uncertainties that obscure their periodic nature. Examples of two passing targets from these populations can be seen in Figure 7.

\section{Application to PTF Data}

Template fitting techniques were applied to the selections of PTF flare candidates described in Section 3. To reduce processing times, each target was fit first for quiet and flare templates, allowing $\Delta \ell_{f q}$ to be calculated prior to RR Lyrae fitting. To remain in the pool of flare candidates, a target must have both a strong $\Delta \ell_{f q}$ and $\Delta \ell_{f r}$. As such, only targets having a $\Delta \ell_{f q}$ metric exceeding the identified $1 \%$ contamination threshold of 10.13 (See Section 7.3) are fit with RR Lyrae templates.

After fitting, we identified a recurrent issue with the data in which an apparent error in the zero-point calculation of PTF's automated photometry routine would induce a large amplitude flux enhancement for a small number of consecutive processed 


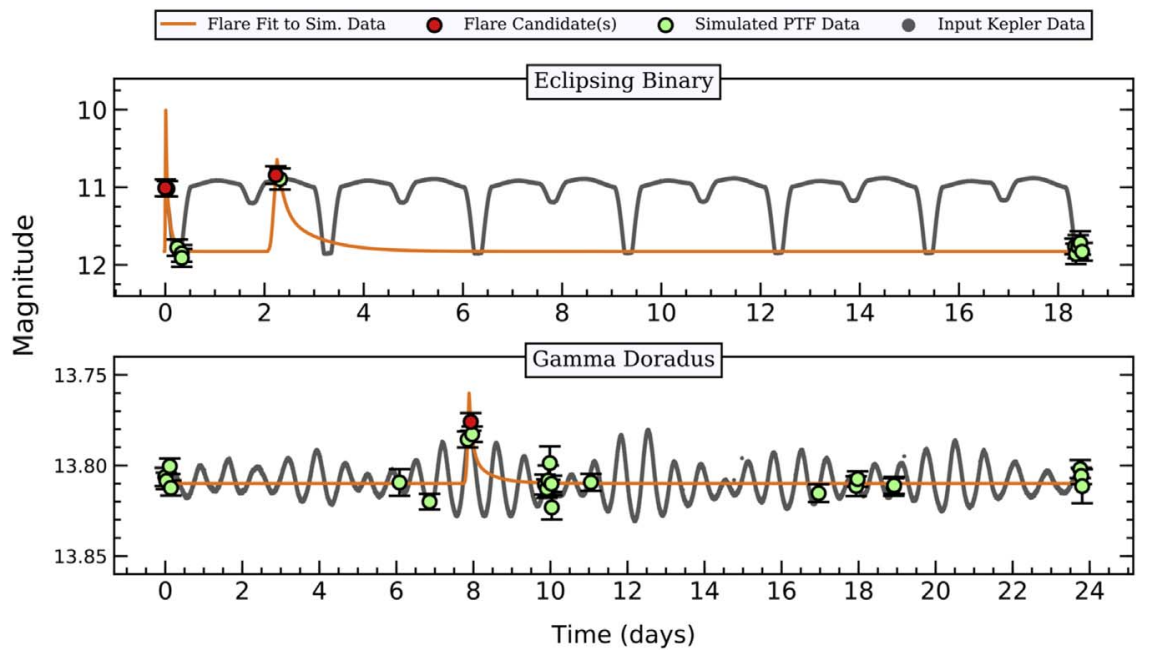

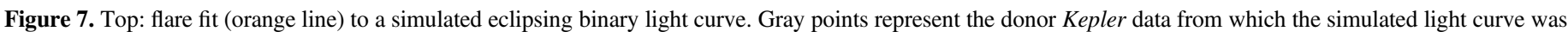

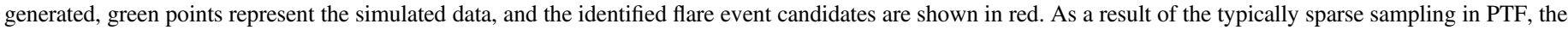

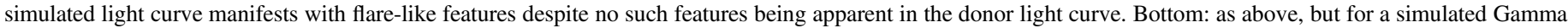

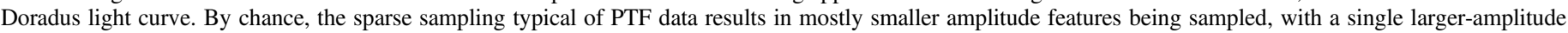

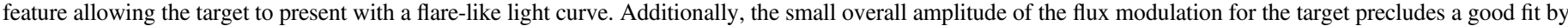
RR Lyrae templates. Combined, these factors allow the target to pass into the regime of likely flare candidates.

frames. This contributed flare-like enhancements to the light curves of any targets in those processed images, and would occasionally allow targets to manifest with passing fit metrics; even though the enhancements may not be fit especially well by a flare template, a massive amplitude event in an otherwise quiet light curve is likely to result in comparatively much worse RR Lyrae and quiet fits. To remedy this issue, in the full set of queried light curves, we counted the number of times that each processed image resulted in a $2.5 \sigma_{\text {med }}+$ outlier for a light curve. We then flagged any processed image resulting in both an outlier event for more than $10 \%$ of light curves in which it appears, as well as resulting in at least two outlier events overall (to avoid eliminating single outliers in processed images that appear only a few times in the sample's light curves). For each target light curve containing a flagged observation, the observation is masked and the target is refit. Targets lacking a flare candidate following this process are eliminated from the candidate pool.

Further, we note the presence of nonastrophysical contamination that cannot be easily distinguished by light curve analysis alone. Most commonly, this resulted from the presence of detector "ghosts." As these bright artifacts move across the detector, they induce time-dependent flux enhancements for sources that they overlap. In some cases, these enhancements result in light curve features that are fit very well by our flare template. The true cause of the apparent event is then only verifiable upon inspection of the source images. As such, we carry out manual inspection of source images to verify flare events from targets for TESS follow-up or from the candidates overlapping with the Kepler and SDSS S82 flare samples.

\subsection{PTF Stripe-82 Fit Results}

Of the 236 flaring stars in the Kowalski et al. (2009) Stripe 82 sample, we recover 10 targets $(4.2 \%)$ above the thresholds determined in Section 7.3 using PTF's data, with a single target being recovered in both $g$ and $R$ bands (see Figure 8). Further, we identify an additional 245 flare star candidates in this region for which flare events were not identified in the prior SDSS S82 sample. Considering the similar recovery hurdles between the PTF data and SDSS data, the fact that our approach recovered a similar number of targets overall (236 versus 255, with 10 targets overlapping) is reassuring evidence that the technique is viable for future application to larger populations.

\subsubsection{Flare Luminosities}

For an additional comparison with the results of Kowalski et al. (2009), we estimate peak flare luminosities for each target recovered from the sample of M dwarfs in the SDSS S82 region. When the shape of the flare candidate is not perfectly constrained, the returned best-fit flaring log-likelihood is degenerate, in that there are a range of fit parameters resulting in a model with the same likelihood. To compute reasonable luminosity estimates for identified flare events, we select the lowest-amplitude flare fit that gives the approximate likelihood originally identified by the algorithm (requiring that the $\chi^{2}$ value of the fit using the new amplitude be within $1 \%$ of the original fit's). In many cases, this constrains the amplitude to the brightest point observed for the event. However, when the data include rise phase observations or exclusively slow decay phase observations, this "minimum" amplitude may be larger.

Luminosities are then computed following the general procedure of Kowalski et al. (2009). For each flare event, the target's extinction corrected quiescent flux is subtracted from that of the flare peak. Distances are determined using parallaxes obtained from the Gaia DR2 catalog (Gaia Collaboration et al. 2018, 2016). Any target that is not matched or that has an associated parallax uncertainty larger than $20 \%$ of the measurement is eliminated from the candidate pool for the purpose of evaluating luminosity. The flare flux and distance measures are then converted to a $g$-band or an $R$-band flare luminosity.

To better compare the luminosities of identified flare event candidates with those of prior studies, we compute flare luminosity conversion factors from PTF's $g$ and R filters to those of SDSS's u filter. For this purpose, we follow the results of Kowalski et al. (2019), which concluded that the $\sim 9000 \mathrm{~K}$ blackbody approximation of flare continuum radiation from 

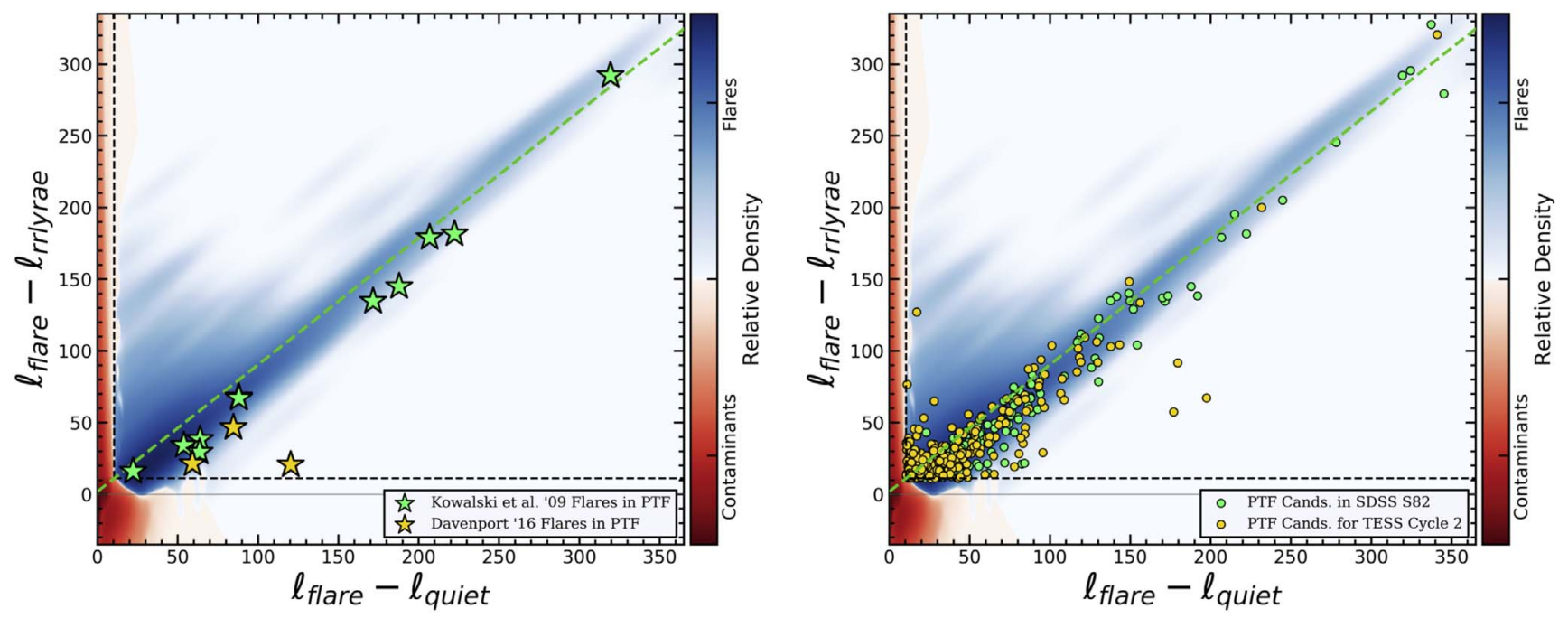

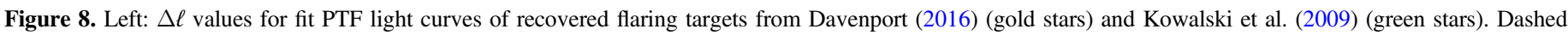

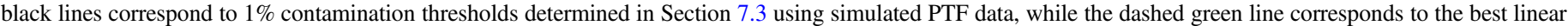

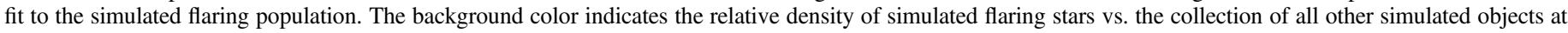

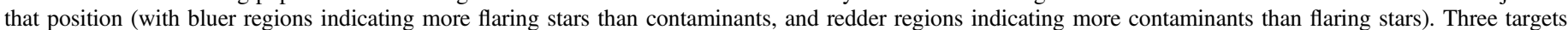

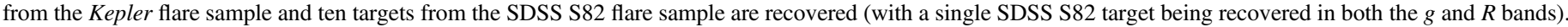

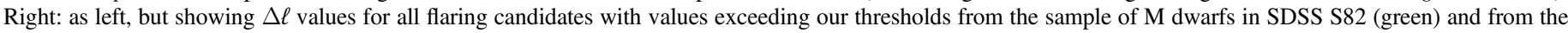
sample of targets considered for follow-up with TESS (gold).

broadband photometry (Hawley et al. 2003) misrepresented the true spectrum of flares in many wavelength regimes. Instead, we compute the fluxes in each filter using the HST-1 flare spectrum provided with Kowalski et al. (2019). The ratio of the flux throughput in any two filters can then be used as an approximate conversion factor for a flare's luminosity between them. Multiplying a computed PTF filter luminosity by the ratio of this $u$-band flux and the respective PTF filter flux provides an approximate corresponding $u$-band luminosity. The determined conversion factors for PTF $g$-band and PTF $R$-band to SDSS $u$-band are 1.125 and 1.623 respectively.

After converting each PTF luminosity using these determined scaling factors, the two filters' samples are combined to produce a single set of $u$-band flare luminosities. For comparison with the SDSS flare sample, the set of luminosities is separated into three spectral type bins: M0-M1, M2-M3, and M4-M6. The resulting histograms (Figure 9) show luminosity distributions that peak at somewhat higher luminosities than those of Kowalski et al. (2009). We use a similar candidacy test, but in bandpasses in which the contrast of flare flux to stellar flux is expected to be much poorer (especially so for PTFs $R$-band), so a higher average recovered flare luminosity is consistent with expectation.

\subsection{PTF Kepler Flare Catalog Fit Results}

Of the 158 M-type stars identified in the Davenport (2016) Kepler Flare Catalog, three targets $(1.9 \%)$ are recovered above thresholds determined in Section 7.3 (see Figure 8). Because Kepler's photometry is generally of significantly higher quality than that of PTF or SDSS, the lower recovery rate for the targets in the Kepler flaring sample compared to that of SDSS S82 may be explained by targets exhibiting flares at a level that was recoverable in Kepler but not with PTF data.

Combined with the results of the SDSS S82 targets overlapping with the Kowalski et al. (2009) sample, this corresponds to recovery of flaring status in $\sim 3 \%$ of tested flaring stars. Though slightly higher than our estimated recovery rate following Section 7.3, this result is consistent

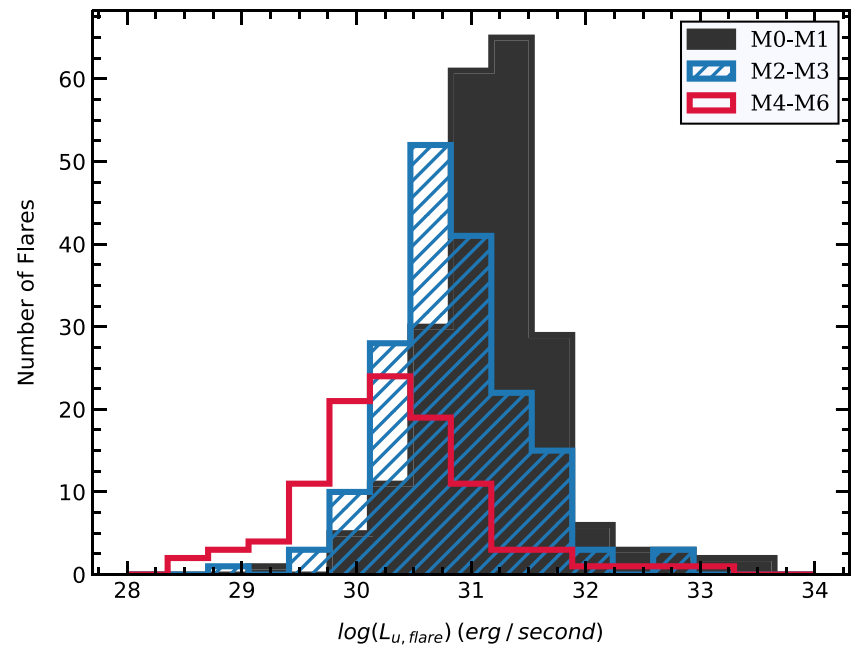

Figure 9. Flare luminosities for strong M-dwarf flare star candidates in PTF data of SDSS Stripe 82, identified using PyVAN. Luminosities have been converted from their native PTF filter to SDSS $u$-band using the ratios of filter convolutions with a flare spectrum from Kowalski et al. (2019). Targets are divided into three spectral type bins for comparison to prior results from Kowalski et al. (2009). Notably, our distributions appear to peak at higher luminosity than those of Kowalski et al. (2009). Because PTF's $g$-band and $R$ band have poorer flare-to-quiescent flux contrast than SDSS's $u$-band, a higher typical flare luminosity for recovered flare events is in line with expectation.

with the expectation of a reduced recovery rate in our simulations due to the smaller average number of observations in our simulated data.

\subsection{PTF TESS Follow-up Candidate Fit Results}

Applying our techniques to the sample of targets for possible TESS follow-up results in an initial sample of $170 \mathrm{~g}$-band candidates and $255 R$-band candidates with $\Delta \ell$ values exceeding our thresholds (see Figures 8 and 10). We approximate a spectral type for each passing target by converting PS1 colors to $B-V$ and $V-I_{C}$, again using color 


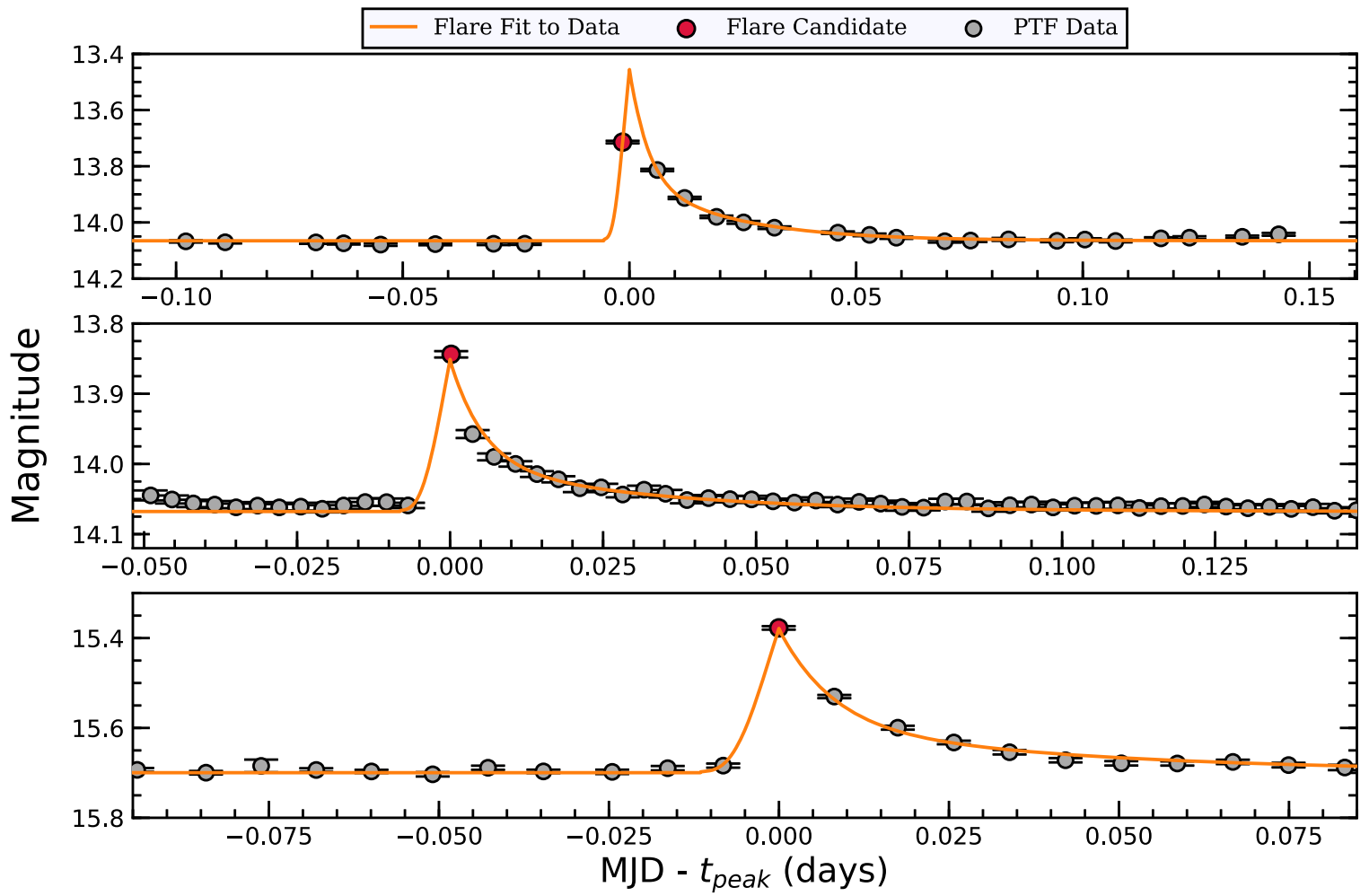

Figure 10. Three strong flare event candidates on M-type stars found in the TESS follow-up sample, identified using PyVAN. The orange line is the determined best flare template fit to the PTF observations (gray points), with the candidate flare peak observation in red. Though the middle and bottom events are fairly well constrained by observations, the top event allows a sizeable range of template parameters that correspond to approximately the same likelihood. Notably, peaks both before or after the candidate observation can result in reasonable fits for this target. The presented fit is the lowest-amplitude event producing a comparable likelihood to the overall best fit (see Section 8.1.1).

Table 2

Candidate G-type Stars Identified for TESS Follow-up

\begin{tabular}{lccccc}
\hline \hline R.A. & Decl. & $\Delta \ell_{f q}$ & $\Delta \ell_{f r}$ & Est. Spectral Type & PTF Filter \\
\hline 85.10256062 & -6.45354963 & 177.0 & 57.4 & G5V & R \\
130.0539418 & 21.11539871 & 95.5 & 29.1 & G6V & R \\
110.8485918 & 26.54241250 & 43.6 & 43.5 & G3V & R \\
190.6555490 & 29.03659331 & 93.3 & 78.3 & G1V & g \\
\hline
\end{tabular}

Notes. Selection of candidate G-type stars identified in PTF data for possible follow-up with TESS. These targets' $\Delta \ell_{f q}$ and $\Delta \ell_{f r}$ values place them well above the identified $1 \%$ contamination thresholds (see Section 7). The process for spectral type estimation is explained in Section 8.3. Here, $m_{0}$ refers to each target's fit quiescent magnitude in the given PTF filter.

transformations from Tonry et al. (2012) and assigning spectral types for these colors based on corresponding values from Pecaut \& Mamajek (2013). Of the full sample, we select a small set of G-type candidates as targets of particular interest for follow-up with high-cadence TESS observations. In addition to requiring passing $\Delta \ell$ values, we require that the images resulting in each targets candidate event is clear of ghosts, halos, or other contributors of possible flux enhancements. Further, because flares on G-type stars have lower contrast with their stellar flux on average (compared to M-type flares) and thus manifest as less statistically significant outliers in our photometry, we require corroboration of flare-like behavior with light curves from the Catalina Surveys (Drake et al. 2009). Specifically, we require at least a single clear outlier observation upon visual inspection of the Catalina Surveys' data. Ultimately, there are four targets meeting this criterion (see Table 2 and Figure 11).

\section{Future Applications}

PyVAN is well-suited to searches for flares (or other variables) in the data of a number of upcoming or recent missions. In the case of LSST data, for instance, PyVAN could serve as a complement to the ANTARES system, which does not currently accommodate flare detection (Narayan et al. 2018).

Though designed for application to sparse ground-based data, PyVAN can also be applied to higher-quality data of space-based surveys (e.g., TESS and Kepler). Applying the software as designed for PTF, stars with flare enhancements in excess of any starspot modulation can be easily separated from those of common contaminants. Efficiency for this use could be improved by application of a detrending routine to candidate light curves before flare fitting (e.g., to flatten spot modulation) and by tuning parameter bounds used in fitting to better utilize the significant constraints provided by such data. 


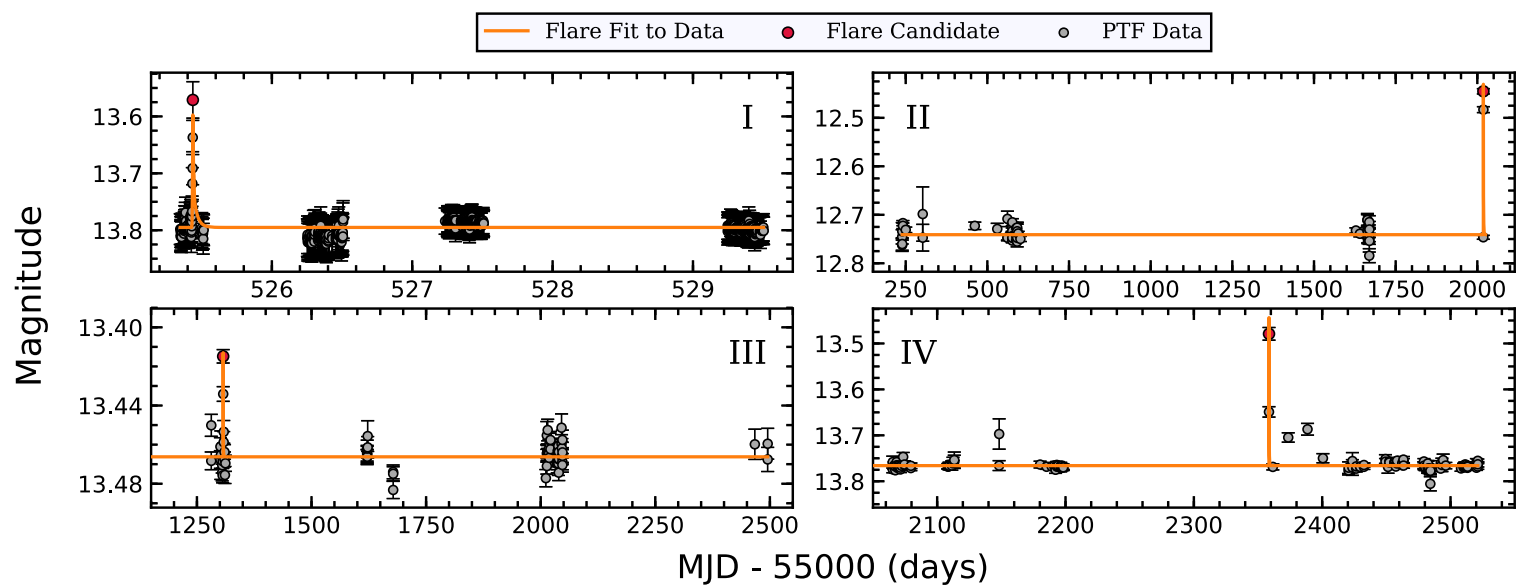

Figure 11. PTF light curves for candidate flaring G-dwarfs with overlaid flare template fit (orange line). The number in the inner corner of each plot indicates the corresponding row in Table 2.

\section{Summary}

We have presented PyVAN, a publicly available template optimization software especially suited to aiding in the recovery of the flaring status of stars from time-domain surveys. PyVAN uses nonlinear optimization algorithms to fit light curve templates of flaring, RR Lyrae, and quiet stars to candidate light curves. Comparing the best odds that each template produced the observed data, PyVAN is able to isolate strong flare star candidates that are also unlikely to be explained by other astrophysical phenomena.

Applying PyVAN to simulated data of comparable signal-tonoise ratio and sampling to that of PTF, we have shown that the technique is capable of filtering out the most pervasive contaminants found in prior samples of flare stars, while recovering a safe population of flaring candidates. In these tests, PyVAN was able to identify $82 \%$ of flare events that we determined to be recoverable. We further estimate from these results an overall flare star recovery rate of $1.5 \%$ for data of similar quality. In application to a PTF sample of $\mathrm{M}$ dwarfs from Stripe 82 and the Kepler field, we have demonstrated PyVAN's ability to produce a sample of flaring candidates that is consistent with previous literature. We have recovered the flaring status of 10 M-dwarf stars from the sample of 236 flaring $\mathrm{M}$ dwarfs $(4.2 \%)$ reported in Kowalski et al. (2009), as well as three from a subsample of 158 flaring $M$ dwarfs (1.9\%) taken from the full sample reported in Davenport (2016). For a combined recovery rate of around $3 \%$, these results are consistent with the speculation that the recovery rate approximated from testing on simulated data was reduced by a number of deficiencies in the simulated sample. Though rates of a few percent may seem to imply a low recovery efficiency, the stochastic nature of flare events combined with time-limited survey data should be expected to place a similarly small upper limit on the rate of recovery that is possible. Searching the light curves of other M dwarfs in Stripe 82, we recover an additional 245 flaring candidates for which flare events were not identified in the Kowalski et al. (2009) sample. Applying our technique to a sample of PTF targets for possible TESS follow-up, we identify four candidate G-type flare stars. Finally, we note potential future applications of the PyVAN software to a number of recent and upcoming missions.

By utilizing PyVAN, or processes implementing similar techniques, samples of probable flaring stars can be produced with significantly diminished rates of contamination.
Mitigating current contamination helps to both improve the efficacy of flare population statistics and to maximize the value of observations with next-generation observatories.

We thank our referee for providing feedback that helped to improve the content and clarity of this manuscript. We also acknowledge support from a Research Corporation Scialog grant.

This paper is based on observations obtained with the Samuel Oschin Telescope and the 60 inch Telescope at the Palomar Observatory as part of the Palomar Transient Factory project, a scientific collaboration between the California Institute of Technology, Columbia University, Las Cumbres Observatory, the Lawrence Berkeley National Laboratory, the National Energy Research Scientific Computing Center, the University of Oxford, and the Weizmann Institute of Science.

The Intermediate Palomar Transient Factory project is a scientific collaboration among the California Institute of Technology, Los Alamos National Laboratory, the University of Wisconsin, Milwaukee, the Oskar Klein Center, the Weizmann Institute of Science, the TANGO Program of the University System of Taiwan, and the Kavli Institute for the Physics and Mathematics of the Universe.

The CSS survey is funded by the National Aeronautics and Space Administration under grant No. NNG05GF22G issued through the Science Mission Directorate Near-Earth Objects Observations Program. The CRTS survey is supported by the U.S. National Science Foundation under grants AST-0909182 and AST-1313422.

\section{ORCID iDs}

Kellen D. Lawson (i) https://orcid.org/0000-0002-6964-8732 John P. Wisniewski ib https://orcid.org/0000-0001-9209-1808 Eric C. Bellm (1) https://orcid.org/0000-0001-8018-5348 Adam F. Kowalski (iD https://orcid.org/0000-0001-7458-1176

\section{References}

Abazajian, K. N., Adelman-McCarthy, J. K., Agueros, M. A., et al. 2009, ApJS, 182, 543

Bellm, E. C., Kulkarni, S. R., Graham, M. J., et al. 2019, PASP, 131, 018002 Blažko, S. 1907, AN, 175, 325

Borucki, W. J., Koch, D., Basri, G., et al. 2010, Sci, 327, 977

Dark Energy Survey Collaboration, Abbott, T., Abdalla, F. B., et al. 2016, MNRAS, 460, 1270

Davenport, J. R., Hawley, S. L., Hebb, L., et al. 2014, ApJ, 797, 122 
Davenport, J. R. A. 2016, ApJ, 829, 23

Debosscher, J., Blomme, J., Aerts, C., \& De Ridder, J. 2011, A\&A, 529, A89

Drake, A. J., Djorgovski, S. G., Mahabal, A., et al. 2009, ApJ, 696, 870

Fuhrmeister, B., Lalitha, S., Poppengaeger, K., et al. 2011, A\&A, 534, 133

Gaia Collaboration, Brown, A. G. A., Vallenari, A., et al. 2018, A\&A, 616, A1

Gaia Collaboration, Prusti, T., de Bruijne, J. H. J., et al. 2016, A\&A, 595, A1

Green, G. M., Schlafly, E. F., Finkbeiner, D., et al. 2018, MNRAS, 478, 651

Hawley, S. L., Allred, J. C., Johns-Krull, C. M., et al. 2003, ApJ, 597, 535

Hawley, S. L., Davenport, J. R. A., Kowalski, A. F., et al. 2014, ApJ, 797, 121

Hawley, S. L., \& Pettersen, B. R. 1991, ApJ, 378, 725

Hilton, E. J. 2011, PhD thesis, Univ. Washington

Howard, W. S., Tilley, M. A., Corbett, H., et al. 2018, ApJL, 860, 30

Juric, M. 2012, LSD: Large Survey Database framework, Astrophysics Source Code Library, ascl:1209.003

Ivezić, Ž., Kahn, S. M., Tyson, J. A., et al. 2019, ApJ, 873, 111

Kinemuchi, K., Smith, H. A., Woźniak, P. R., McKay, T. A. \& ROTSE Collaboration 2006, AJ, 132, 1202

Kirk, B., Conroy, K., Prša, A., et al. 2016, AJ, 151, 68

Kowalski, A. F., Hawley, S. L., Hilton, E. J., et al. 2009, AJ, 138, 633

Kowalski, A. F., Hawley, S. L., Holtzman, J. A., Wisniewski, J. P., \& Hilton, E. J. 2010, ApJL, 714, 98

Kowalski, A. F., Hawley, S. L., Wisniewski, J. P., et al. 2013, ApJS, 207, 15

Kowalski, A. F., Wisniewski, J. P., Hawley, S. L., et al. 2019, ApJ, 871, 167

Lacy, C. H., Moffett, T. J., \& Evans, D. S. 1976, ApJS, 30, 85

Law, N., Fors, O., Ratzloff, J., et al. 2015, PASP, 127, 234

Law, N. M., Kulkarni, S. R., Dekany, R. G., et al. 2009, PASP, 121, 1395

Lomb, N. R. 1976, Ap\&SS, 39, 447
Loyd, R. O. Parke, France, K., Youngblood, A., et al. 2018a, ApJ, 867, 71

Loyd, R. O. Parke, Shkolnik, E. L., Schneider, A. C., et al. 2018b, ApJ, 867, 70

Magnier, E. A., Schlafly, E., Finkbeiner, D., et al. 2013, ApJS, 205, 20

Moffett, T. J. 1974, ApJS, 29, 1

Narayan, G., Zaidi, T., Soraisam, M. D., et al. 2018, ApJS, 236, 9

Nemec, J. M., Cohen, J. G., Ripepi, V., et al. 2013, ApJ, 773, 181

Newville, M., Stensitzki, T., Allen, D. B., et al. 2016, Lmfit: Non-Linear Least-

Square Minimization and Curve-Fitting for Python, v0.9.12, Astrophysics

Source Code Library, ascl:1606.014

Nutzman, P., \& Charbonneau, D. 2008, PASP, 120, 317

Pecaut, M. J., \& Mamajek, E. E. 2013, ApJS, 208, 9

Richards, J. W., Starr, D. L., Butler, N. R., et al. 2011, ApJ, 733, 10

Ricker, G. R., Winn, J. N., Vanderspek, R., et al. 2015, JATIS, 1, 014003

Scargle, J. D. 1982, ApJ, 263, 835

Schmidt, S. J., Shappee, B. J., van Saders, J. L., et al. 2019, ApJ, 876, 115

Segura, A., Walkowicz, L. M., Meadows, V., Kasting, J., \& Hawley, S. 2010, AsBio, 10, 751

Sesar, B., Ivezić, Ž., Grammer, S. H., et al. 2010, ApJ, 708, 717

Shappee, B. J., Prieto, J. L., Grupe, D., et al. 2014, ApJ, 788, 48

Silverberg, S. M., Kowalski, A. F., Davenport, J. R., et al. 2016, ApJ, 829, 129

Stetson, P. B. 1996, PASP, 108, 851

Storn, R., \& Price, K. 1997, J. Global Optim., 11, 341

Tonry, J. L., Stubbs, C. W., Lykke, K. R., et al. 2012, ApJ, 750, 99

Vida, K., \& Roettenbacher, R. M. 2018, A\&A, 616, A163

Walkowicz, L. M., Basri, G., Batalha, N., et al. 2011, AJ, 141, 50

Yang, H., Liu, J., Gao, Q., et al. 2017, ApJ, 849, 36

Zinn, J. C., Kochanek, C. S., Kozlowski, S., et al. 2017, MNRAS, 468, 2189 